Results Case 1: A 28 year old HIV-positive man presented 96 hours after taking a deliberate overdose of $40 \times 400 \mathrm{mg}$ raltegravir tablets. He developed mild symptoms of diarrhoea, abdominal cramps and a sore chest. Results post-overdose: electrolytes and renal function: normal; liver function tests: ALT 58, others normal; creatinine kinase 67; haematology: normal; therapeutic drug monitoring (TDM) results: raltegravir not detected 96 hours post overdose. There were no serious complications. Case 2: A 52 year old HIV-positive man presented 24 hours after taking a deliberate overdose of $18 \times$ Truvada (tenofovir disaproxil fumarate/emtricitabine). He had no symptoms related to the overdose. Results post-overdose: urinalysis: normal; electrolytes and renal: phosphate 0.75, creatinine 94; TDM: 24 hours: 207 ng/ml; 48 hours: $80 \mathrm{ng} / \mathrm{ml}$; 72 hours: $16 \mathrm{ng} / \mathrm{ml}$; 192 hours: 2 $\mathrm{ng} / \mathrm{ml}$. There were no serious complications.

Discussion/conclusion The patients in our case series showed few side effects and no serious sequelae as a result of their overdose. There seems to be little guidance available to guide management of such cases.

\section{P059 A REVIEW OF SEXUALLY ACQUIRED PROCTITIS: AN ARRAY OF SYMPTOMS, INVESTIGATIONS AND TREATMENTS}

Elizabeth Nicol, Jonathan Shaw*, Christopher Ward. The Hathersage Integrated Contraception, Sexual Health and HIV Service, Manchester, UK

10.1136/sextrans-2016-052718.113

Introduction With recent lymphogranuloma veneruem (LGV) and Shigella outbreaks amongst men-who-have-sex-with-men (MSM), proctitis has become a prominent clinical issue. There is no UK guideline regarding proctitis management but guidance is available from IUSTI and CDC.

Objectives To review our proctitis cases and generate a clinic policy to standardise practice.

Methods Casenotes coded C4NR between 01/01/14-31/12/15 were reviewed with data collated and analysed via Microsoft Excel.

Results 100 care episodes were reviewed (92 patients, 6 attended twice and 1 thrice). All patients were male; 83 homosexual, 8 bisexual and 1 heterosexual. 67 patients were White British, 31 were HIV positive. Median age was 29 years (range $=18-62$ ). Presenting symptoms were varied with rectal pain (58), discharge (54), and bleeding (44) most common. Proctoscopy in 82 cases found varying signs (32 discharge, 24 oedema, 25 contact bleeding, 10 ulceration). Microscopy was diagnostic of proctitis in 39/ 84 (46.4\%) patients. Physician-requested investigations were:

\begin{tabular}{lll} 
Abstract P059 Table 1 & Sexually acquired proctitis \\
\hline Test & Performed & Positive results \\
\hline Chlamydia trachomatis TMA & 100 & 14 (including 6 LGV) \\
Neisseria gonorrhoea TMA & 100 & 28 \\
Gonococcal culture & 98 & 24 \\
Herpes Simplex PCR & 30 & 9 \\
Treponema pallidum PCR & 27 & 1 \\
Treponemal serology & 93 & 5 \\
Stool culture & 13 & 4 \\
\hline
\end{tabular}

Treatment at initial visit was predominantly doxycycline-based (99/100), with course length varying from 7-21 days.
Concurrent therapies were influenced by clinical findings and reported contacts; predominantly ceftriaxone (53), azithromycin (31), and aciclovir (19).

Conclusions Gonorrhoea incidence was high (28\%), as was herpes when requested (30\%). Updated clinic policy for all proctitis patients includes requesting Herpes Simplex PCR and presumptive treatment for gonorrhoea.

\section{P060 ARE WE 'SPOTTING THE SIGNS?'}

Arnold Fernandes*, Mary-Anne Bailey, Ameeka Thompson, Kate Horn. Royal United Hospital, Bath, UK

\subsection{6/sextrans-2016-052718.114}

Background/introduction In 2014, BASHH/Brook piloted a proforma for identifying risks of child sexual exploitation in sexual health settings in the light of cases of child sexual exploitation identified nationally. Use of the pro-forma was promoted. The form was introduced in our unit in October 2014 following discussion. This replaced the 'under-16's risk-assessment,' used previously.

Aim(s)/objectives To assess as to whether the pro-forma was being used overall, with in-depth analysis of key components of the document.

Methods Retrospective note audit between 01/04/15-30/09/215 conducted. 44 attendees under 18, identified (17 male, 27 female)

Results The form was used in 39/44 (88.6\%). Assessment of Fraser competence was documented in 34/42 (81\%); 2 attendees were over 16 . There was documentation that 'confidentiality clause' was discussed in $37 / 44$ (84.1\%). Age of partner was documented in 35/42 (83.3\%); 2 patients had never had sex and hence were excluded. Name of social worker was documented in $7 / 13(53.8 \%)$ attendees who had indicated they had one. 31 attendees had no social worker. Professional analysis was completed in 16/44 (36.4\%). However, a further 10 notes had comments documented, which increased completion rate to $59.09 \%$. Discussion/conclusion After initial concerns raised by Staff about time taken to complete the form and the qualitative nature of information included in the form, this was incorporated into most consultations including under-18 attendees. Documentation on most key aspects of the pro-forma was generally good, with room for improvement. A feed-back session for staff combined with a further dedicated teaching session on safe-guarding is organised to improve this.

\section{P061 SAFETY OF SINGLE DOSE GENTAMICIN COMPARED WITH MULTIPLE DOSE REGIMENS}

${ }^{1}$ Rachel Hayward*, ${ }^{1}$ Jan Harding, ${ }^{1}$ Robert Molloy, ${ }^{3}$ Lucy Land, ${ }^{1}$ Kate Longcroft-Neal, ${ }^{2}$ Dave Moore, 'Jonathan Ross. ${ }^{1}$ Whittall Street Clinic, Birmingham, UK; ${ }^{2}$ University of Birmingham, Birmingham, UK; ${ }^{3}$ Birmingham City University, Birmingham, UK

\subsection{6/sextrans-2016-052718.115}

Background Traditionally, gentamicin is given eight hourly, guided by drug levels. Several studies have shown that singledaily dosing of gentamicin offers an equal, if not improved, toxicity profile compared to traditional dosing. Single one-off dose gentamicin has been suggested as treatment for gonorrhoea, but its safety has not been reviewed.

Aim Systematically review the frequency and type of adverse events associated with a single dose of intravenous or 
intramuscular gentamicin in adults, for any indication, in studies where a comparator was available.

Methods A review protocol was developed and registered (PROSPERO: CRD42013003229). Studies were eligible for review if they; recruited participants $\geq 16$ years old, used gentamicin intramuscularly or intravenously as a single one-off dose, compared gentamicin to another medication or placebo, and if adverse events were monitored. We searched MEDLINE, EMBASE and other relevant databases. Risk of bias was assessed in included studies.

Results 12,116 records were identified. After removal of duplicates, screening of title/abstracts for relevance and independent selection of full texts by two reviewers, 20 studies were included. 3589 participants were analysed across all studies, 2042 received a single one-off dose of gentamicin (doses ranged from $1 \mathrm{mg} / \mathrm{kg}$ - $280 \mathrm{mg}$ ). Reversible nephrotoxicity/creatinine rise was reported in 37 cases, with one case of irreversible renal impairment. There were three cases of ototoxicity, with similar frequency reported in the comparator group. A meta-analysis was not possible due to heterogeneity. Reporting of adverse events was poor in the majority of studies.

Discussion Adverse events with single dose gentamicin are infrequent.

\section{P062 A RETROSPECTIVE AUDIT OF HEPATITIS B MANAGEMENT IN THE GU CLINIC}

Elizabeth Nicol*, Chris Ward. The Hathersage Integrated Contraception, Sexual Health and HIV service, Manchester, UK

\subsection{6/sextrans-2016-052718.116}

Background/introduction Background/introduction: Hepatitis B (HBV) is a sexually transmitted infection commonly diagnosed in GU settings. We routinely test for $\mathrm{HBV}$ in high risk patients such as men who have sex with men, sex workers and those from high prevalence areas.

Aim(s)/objectives A retrospective audit was undertaken assessing whether patients diagnosed with $\mathrm{HBV}$ are being managed in accordance with BASHH guidance and whether changes made following a previous audit were implemented successfully.

Methods Patients newly diagnosed with HBV over an 18 month period up to $31 / 8 / 15$ had their records reviewed. Data was collected on demographics, investigations, initial management and follow up.

Results 31 patients were included in the audit and their care compared to the 2008 national auditable standards. Median age was 28(range 16-46). 20 (64.5\%) were male and $11(35.5 \%)$ female. $29(93.5 \%)$ identified as heterosexual, $2(6.5 \%)$ as homosexual. 11 (35.5\%) were of African descent. Only 6\% of patients were provided with written information on HBV transmission and outcomes and 68\% had documented partner notification. 95\% had liver function tests performed post diagnosis, $97 \%$ had clear long term management plans documented and $87 \%$ were offered appointments with hepatology(compared to $67 \%$ in the previous audit).

Conclusion Clinicians were failing to provide written information about HBV following diagnosis as information leaflets were not available in clinic. $87 \%$ of patients received verbal health advice but this still falls short of national standards. A scheme giving cards with QR codes linking to approved patient information is being trialled. Improved referral pathways have resulted in more patients having specialist review.

\section{P063 STAFF, ASSOCIATE SPECIALIST AND SPECIALTY (SAS) DOCTORS' NATIONAL AUDIT ON THE MANAGEMENT OF GONORRHOEA IN THE UK}

${ }^{1}$ Helen Mullan*, ${ }^{2}$ Carlos Oroz, ${ }^{3}$ Jane Richards, ${ }^{4}$ John Lee. ${ }^{1}$ Central London Community Healthcare NHS Trust, London, UK; ${ }^{2}$ Chalmers Sexual Health Centre, Edinburgh, UK; ${ }^{3}$ Newcastle Hospitals NHS Foundation Trust, Newcastle-upon-Tyne, UK; ${ }^{4}$ Josephine Butler Centre, Wakefield, UK

\subsection{6/sextrans-2016-052718.117}

Background/introduction The British Society for Sexual Health and HIV (BASHH) revised United Kingdom national guideline for the management of gonorrhoea in adults, 2011, identified five auditable outcome measures. The UK National Guideline for Gonorrhoea Testing, Clinical Effectiveness Group, BASHH, 2012, suggested a further three.

Aim(s)/objectives The aim was to audit national management of gonorrhoea against the standards recommended in these two documents. Only SAS doctors were eligible to participate.

Methods SAS doctors, whose details were registered on the SAS database, were invited to contribute. All clinics were asked to designate a Local Co-ordinator who would register that clinic and allocate patients to the other participating doctors. Results for the individual clinics were sent to the Local Co-ordinator for dissemination. All information was submitted via a secure online link. Data from forty patients was requested.

Results 3233 cases were submitted from 78 centres by 168 doctors. $68 \%$ cases were male and 44\% were MSM. $83 \%$ received first line treatment. $97 \%$ were tested or treated for CT. $41 \%$ offered written information on GC. Culture attempted in $86 \%$ of those GC NAAT positive. Sensitivity testing performed on 94\% culture positive. Supplementary testing performed on $61 \%$ throat and $60 \%$ rectal NAAT reactive. TOC performed on $61 \%$; $41 \%$ of these within 2 weeks. Partner notification was done in 92\% cases.

Discussion/conclusion $83 \%$ patients received first-line treatment. A reason was provided for $11 \%$ treated with other regimens. The number offered written information was low at $41 \%$. Results about supplementary testing were inconsistent. The results for the other outcomes were satisfactory.

\section{P064 DELIVERY OF SEXUAL HEALTH INTERVENTIONS FOR MEN WHO HAVE SEX WITH MEN (HPV VACCINATION AND PREP) MAY BE HINDERED BY THE LACK OF SEXUALITY DISCLOSURE IN PRIMARY CARE}

${ }^{1}$ Samuel Owen*, 1,2Thomas Kurka, 1,2Daniel Richardson. 'Brighton and Sussex Medical School, Brighton, UK; ${ }^{2}$ Brighton \& Sussex University Hospitals NHS Trust, Brighton, UK

\subsection{6/sextrans-2016-052718.118}

Background/introduction Large scale new sexual heath interventions for MSM (HPV vaccination, PrEP) will potentially need to be delivered in primary care as well as sexual health. It is important these services are acceptable to MSM and MSM feel confident to disclose their sexuality.

Methods From November 2015 to February 2016, a paper survey was distributed to MSM attending local sexual health services on acceptability of local services and initial disclosure of sexuality to a healthcare setting. National Student Pride also used an online version of the survey.

Results 1186 MSM were included in the analysis of this survey. The median age was 26.8 years (18-89). 1026/1186 (86.5\%) self-identified as gay, 108/1186 (9.1\%) bisexual and 34/1186 